\title{
The potential of simple, low-cost permanent magnets for flame manipulation in flow fields
}

\begin{abstract}
A method to manipulate propane diffusion flame using low strength permanent magnets has been studied in this work. It involves determining the impact of increasing and decreasing gradient magnetic fields on flame structure and liftoff height as a possible route towards flame stabilization and combustion control. A non-uniform magnetic field was produced by using two low strength permanent magnets mounted on an iron yoke. Propane/air flames with different flow velocities produced by two different burners were subject to the non-uniform field. A "false magnet" is used to extract only the impact of the magnetic force on flames from the buoyance force generated due to the presence of the yoke. For an attached flame, it was observed that, an increasing field increased the height of the flame and this effect was more pronounced at higher flow rates. A decrease in the flame height was observed in the presence of a decreasing gradient field. For a lifted flame, it was observed that the liftoff height was decreased in the presence of an increasing and a decreasing gradient field; this effect appeared to be independent of the burner size. The liftoff height is appeared to be reduced by both the aerodynamic effect and the magnetic field effect of the yoke.
\end{abstract}

Volume 2 Issue I - 2018

\author{
Vikram Ramnath, Kevin M Lyons \\ Mechanical and Aerospace Engineering, North Carolina State \\ University, USA
}

Correspondence: Vikram Ramnath, Mechanical and Aerospace
Engineering, North Carolina State University, Raleigh, NC 27695-79I0 USA; Email vramnat@ncsu.edu

Received: July 28, 2017| Published: January 18, 2018

\section{Introduction}

The effect of electric fields on combustion is well established. However, few studies have been conducted about the effect of magnetic fields on combustion, though the fact that magnetic fields can influence combustion was first realized over one hundred and fifty years ago. As early as 1846, Faraday applied a magnetic field to a flame on a wax taper and observed its tendency to form an equatorial disc. ${ }^{2}$ Para magnetism is the situation where certain materials are attracted by an externally applied magnetic field, and form internal, induced magnetic fields in the direction of the applied magnetic field. Oxygen, an element essential for combustion is considered a paramagnetic substance because the oxygen molecule has two unpaired electrons that spin in the same direction as each other, increasing the magnetic field effect. The products of combustion, in contrast are diamagnetic materials. They are repelled by magnetic fields and form induced magnetic fields in the direction opposite to that of the applied magnetic field. Ueno ${ }^{3}$ also examined the ability of the magnetic field to quench flames. A candle flame between two columnar electromagnets, hollowed out, to enclose the flame was exposed to a field of $1.5 \mathrm{~T}$ in an air gap of 5-10 $\mathrm{mm}$. The flame was quenched soon after the application of the magnetic field. Mizutani et al. ${ }^{4}$ studied the effect of a uniform magnetic field on premixed laminar flames. The structure of the flame was observed using a schlieren system. It was observed that the presence or absence of a magnetic field had no perceptable influence on the flame structure. They inferred that the magnetic field, even ones as high as $5 \mathrm{~T}$ had no effect on the burning velocity as it was dominated by high-speed chemical reactions.

Gillon et al. ${ }^{5}$ studied the behaviour and stability of a laminar methane air flame with an air co-flow for anchored and lifted conditions for different flow regimes under the influence of a uniform field. It was observed that application of the magnetic field reduces liftoff height and that it was dependent on the co-flow air velocity. For the flame and co-flow experiencing an upward increasing magnetic field gradient, it was observed that there was a decrease in the liftoff height. This was due the action of the magnetic gradient which blocked the ambient air at the entrance of the magnet. This reduced the entrainment by the air co-jet, resulting in the reduction of the flame lift height. The use of electromagnets is a major drawback of these investigations because they require significant energy to produce the magnetic fields. Recent studies using lower strength electromagnets studied the properties of lifted flames using an air co-flow. The direct impact of these gradient fields on the flames and the hydrocarbon fuel is greatly diminished due to the paramagnetic nature of the co-flowing air that forms a shield around the diamagnetic combustion products. To fully understand the complex nature of the impact of magnetic fields on flame behavior, the effects of magnetic fields produced by permanent magnets of relatively low strengths are studied as a stepping stone to a possible route towards combustion control and flame stabilization by magnetism. The objective of the current investigation is to determine if gradient magnetic fields produced by relatively low strength permanent magnets could effectively alter the combustion characteristics of a small laminar flame. The study anticipates determining if these low strength magnetic fields can impact a flame outside a relatively narrow sphere of influence. The different properties of the flame such as liftoff height, flame structure of a propane jet flame are investigated.

\section{Experimentation}

The investigation was conducted at the Reacting Flow and Turbulent Jets Laboratory in the MAE department of NC State University. The description of the various components and the general setup used in this investigation is described in the following paragraphs. A picture of the experimental setup is shown in Figure 1. The experimental setup consists of the burner, yoke with the magnets, illuminated scale and the image capture device. The burner, shown in Figure 2, consists of a cylindrical base set on a stand through which a series of PVC fittings have been configured. A gaseous fuel supply line is attached to the central fuel jet. The fuel tube, has an outer diameter of $1.57 \mathrm{~mm}$, made of stainless steel extends from the bottom of the apparatus through the center of the PVC fittings and ends outside the concentric PVC casing. Two different fuel tubes having a $0.81 \mathrm{~mm}$ and $0.55 \mathrm{~mm}$ inner diameter 
are selected for this investigation. Due to a burner length of $125 \mathrm{~mm}$, the gas flow is assumed to be fully developed at the exit of the burner, with a Poiseuille-type velocity distribution. The fuel used, $99.0 \% \mathrm{CP}$ grade propane $\left(\mathrm{C}_{3} \mathrm{H}_{8}\right)$ was supplied from the cylinder. It flows through a Yor-Lok precision flow adjustment needle valve and was monitored using mass flow meter (OMEGA, FMA-A2309) having a range of 0-50 SLPM. The readings were estimated to be accurate within 5\%. This range is sufficient for experiments to be performed over a wide range of jet velocities.

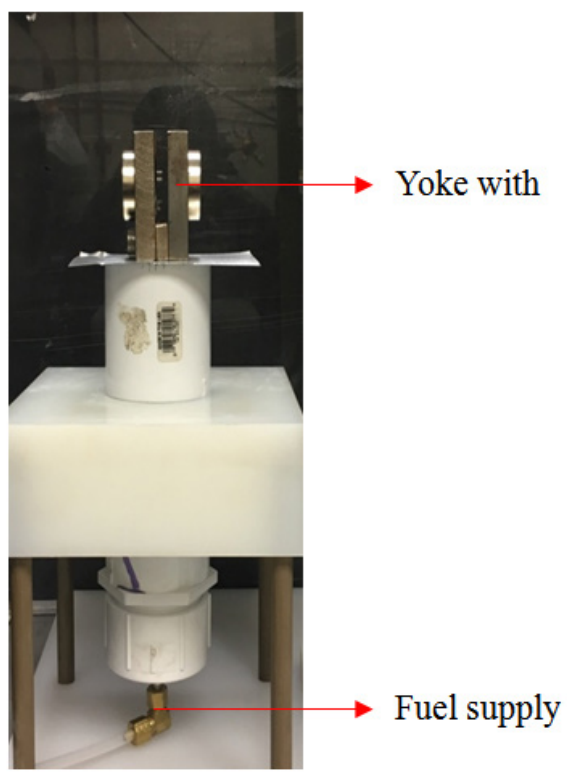

Figure I Experimental Setup.

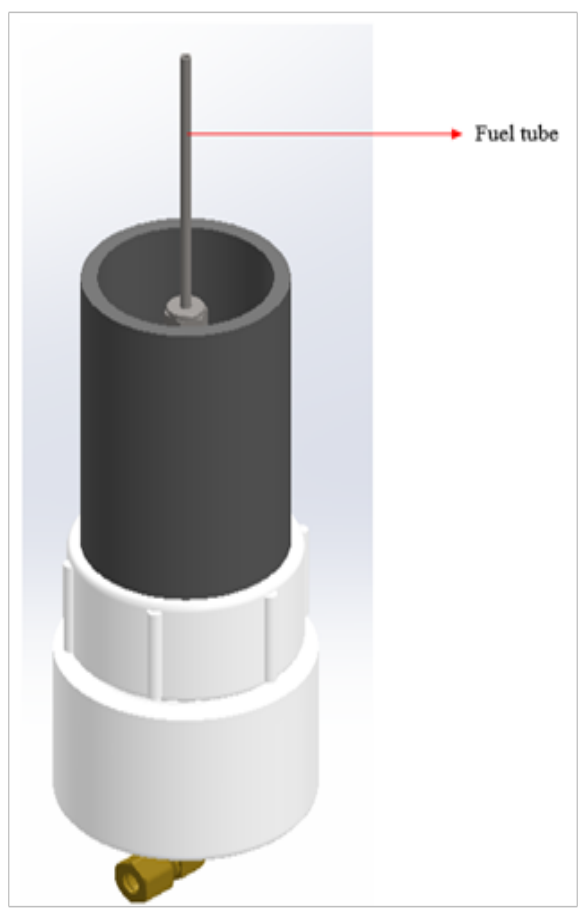

Figure 2 Burner CAD diagram.

In this investigation, N52 grade permanent magnets (DX8C-N52) having a diameter of $37.6 \mathrm{~mm}$ and a depth of $19.05 \mathrm{~mm}$ is used to generate the gradient magnetic field. Two magnets, each of which could produce a pull force of $52.3 \mathrm{Kg}$ towards a steel plate are used in the current investigation. Placing two of these magnets in an iron yoke with a $7.62 \mathrm{~mm}$ air gap, as shown in Figure 3, complete a path for the magnetic flux which enables a magnetic field whose maximum strength at the center is $0.75 \mathrm{~T}$ to be formed between the poles of the magnet (Figure 4). The yoke is designed to be mounted on the fuel jet. The height of the fuel jet is adjusted in a manner that produces an increasing or a decreasing gradient magnetic field as shown in Figure 5. This arrangement is oriented in a manner that is best for image capture. An active cooling arrangement for the magnets was deemed unnecessary as the duration of the individual experimental runs were short which ensured that the temperature of the yoke and the magnets did not reach the Curie temperature. A series analysis of the strength of the magnets after each successive test revealed no significant loss of magnetic strength and thus the assumption that the magnetic field strength remained constant throughout the investigation is supported. A set of experiments were carried out to determine the flame structure, liftoff height and the minimum flow required to sustain a lifted flame. The flame structure test is conducted to determine the impact of magnetic field gradients on the height of the diffusion flame. For a circular-port flame, the flame length does not depend on initial velocity or diameter but, rather, on the initial volumetric flow rate ${ }^{6}$ so; the propane flow rate is varied to produce a flame whose height is within the influence of the magnetic field.

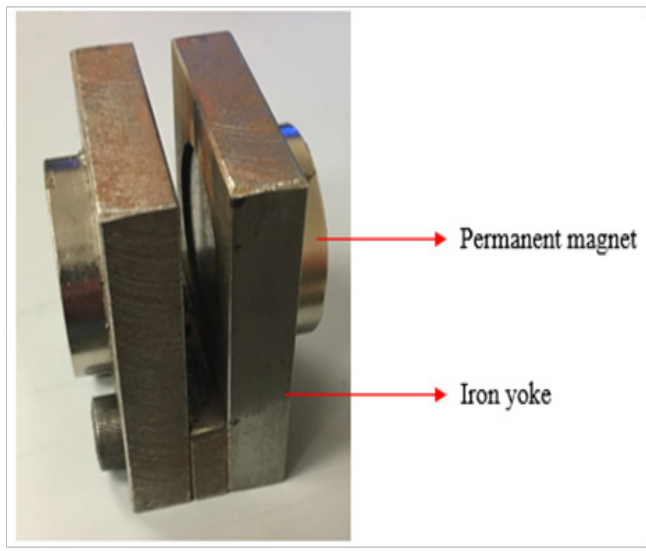

Figure 3 Iron yoke with magnets.

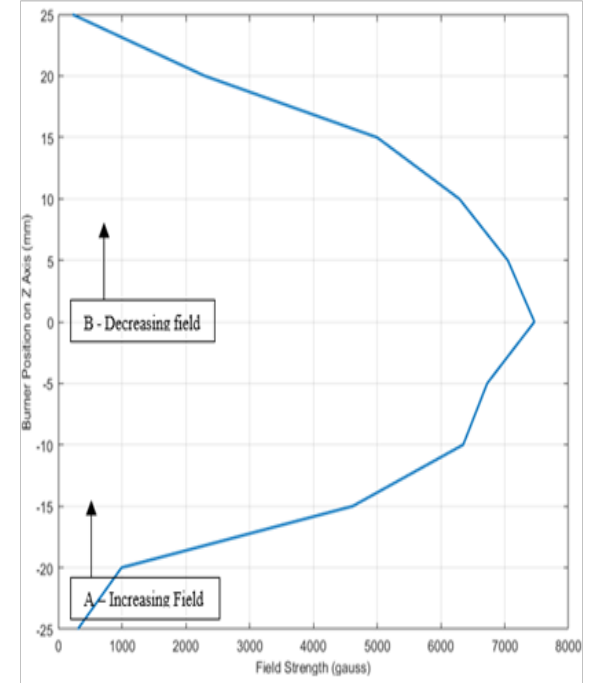

Figure 4 Permanent magnet field strength variation. 


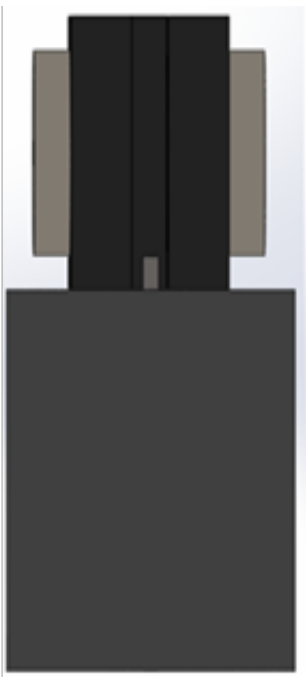

(a)

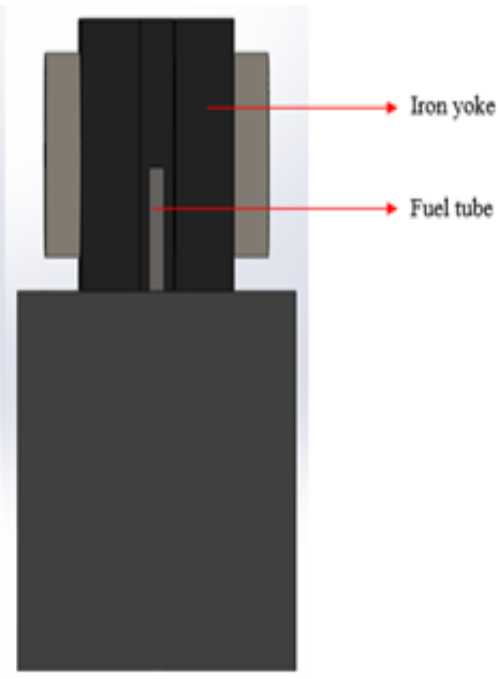

(b)

Figure 5 Comparison of fuel tube position for (a) increasing; (b) decreasing magnetic field.

The variation of the flame liftoff height under the influence of gradient fields is studied. The lifted flame investigation is conducted by varying the propane flow rate as required. Using a yoke to hold the magnets in place gives rise to two different impacts-a dynamic impact on the flow field and a magnetic impact. In this investigation, we consider only the magnetic impact on the flow field. To extract only the impact of the magnetic force on flames from the buoyance force generated due to the presence of the yoke, a yoke made of aluminum possessing the exact geometry of the magnetic yoke is fabricated and all the baseline investigations are done in the presence of the aluminum yoke. This aluminum yoke is called the "false magnet". The flame images were captured using a digital single-lens reflex camera (Nikon Corp, Model: D80), and the flame images were analyzed using in house codes (Figure 6).

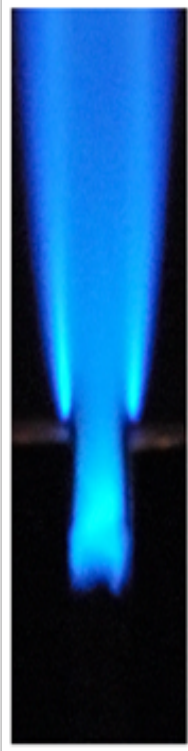

(a)

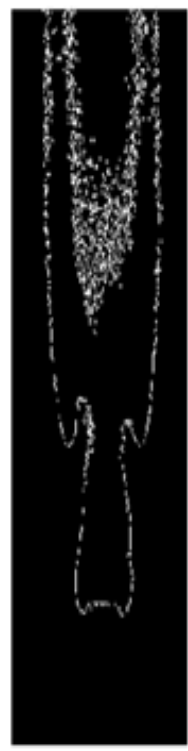

(b)

Figure 6 Comparison of (a) raw image obtained from camera and (b) corresponding image of the flame edge obtained by image processing.

\section{Results}

\section{Increasing gradient field}

The variation in flame height and flame liftoff height in the presence of a gradient magnetic field whose strength was increasing from the burner lip is studied. This data is compared to measurements studied in the absence of a magnetic field. The variation of the flame height in the presence and absence of the gradient magnetic field were used to determine if it had a significant impact on the flame height. The flame height is defined as the perpendicular distance between the burner lip and the tip of the flame. Figure $7 \&$ Figure 8 show the variation in flame height compared in the presence and absence of a gradient field for the $0.55 \mathrm{~mm}$ and $0.81 \mathrm{~mm}$ burner respectively. It is observed that the presence of increasing gradient magnetic causes an increase in the flame height compared to the no field applied case for both the burners. For the $0.55 \mathrm{~mm}$ burner, it is observed that the increase in height is more pronounced for the high flow rates. Also, it can be noted that an overall larger increase in height can be noted for the flame produced by the larger burner. Figure 9 shows the variation in flame liftoff height for the $0.81 \mathrm{~mm}$ burner. It is compared to the flame liftoff height measured in the absence of a magnetic field. It is observed that an increasing field decreases the liftoff height of the flame when compared to the no field applied case. A lifted flame could not be obtained for the low flow rates.

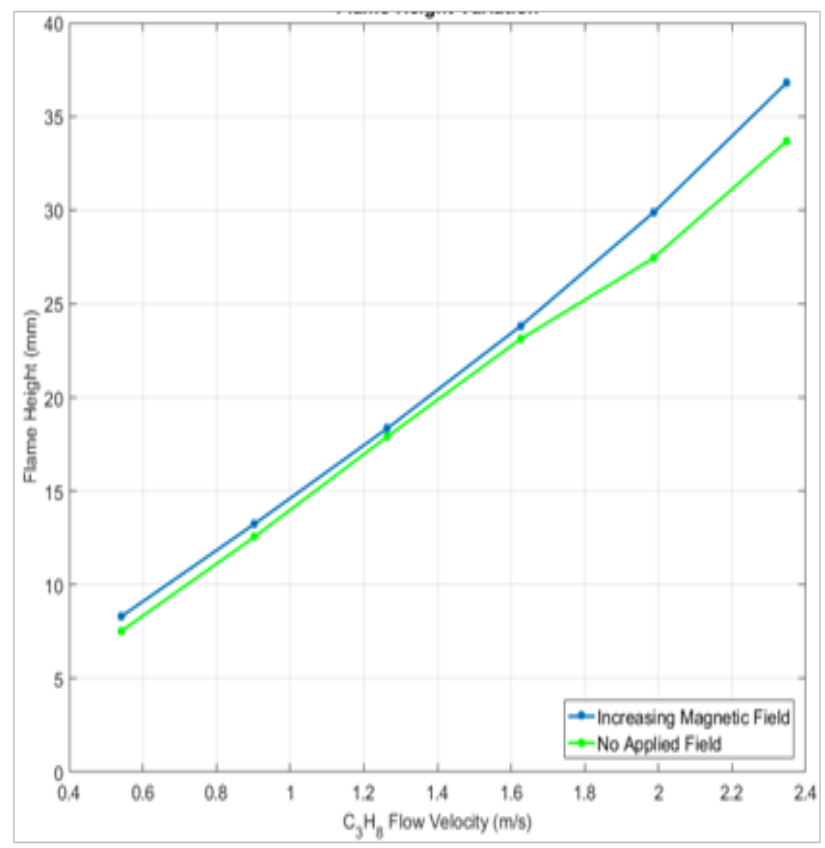

Figure 7 Flame height variation in an increasing gradient field for the 0.55 mm burner.

\section{Decreasing gradient field}

The variation in flame height and flame liftoff height in the presence of a gradient magnetic field whose strength was decreasing from the burner lip is studied and compared to measurements in the absence of a magnetic field. Figure 10 \& Figure 11 show the variation in flame height for two different burner sizes and is compared to the flame height measured in the absence of a magnetic field. It is observed that the presence of decreasing gradient magnetic causes a decrease in the flame height compared to the no field applied case for the $0.55 \mathrm{~mm}$ burner. It has a negligible impact on the larger burner. It is observed 
that a change in burner size influences the change in flame height. This shows that the impact of decreasing gradient field on flame height is dependent of the burner size. Figure 12 shows the flame liftoff height variation for the burner with a $0.55 \mathrm{~mm}$ diameter. It is compared to the flame liftoff height measured in the absence of a magnetic field. It is observed that the presence of a decreasing gradient magnetic causes a decrease in the flame height. Increase in flow velocity increases the reduction in liftoff height. The reduction in height appears dependent on the fuel flow velocity. Figure 13 shows the variation in flame liftoff height for the $0.81 \mathrm{~mm}$ burner. It is compared to the flame liftoff height measured in the absence of a magnetic field. It is observed that a decreasing gradient magnetic causes a decrease in the flame liftoff height. The reduction in height appears to be fairly constant which shows that this effect is independent of the fuel flow velocity for the larger burner.

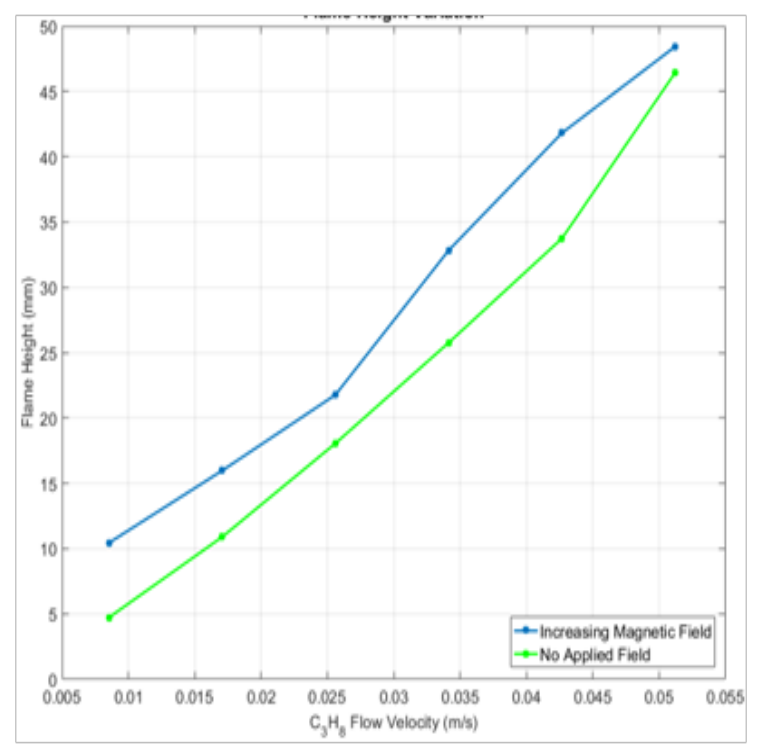

Figure 8 Flame height variation in an increasing gradient field for the $0.8 \mathrm{I}$ $\mathrm{mm}$ burner.

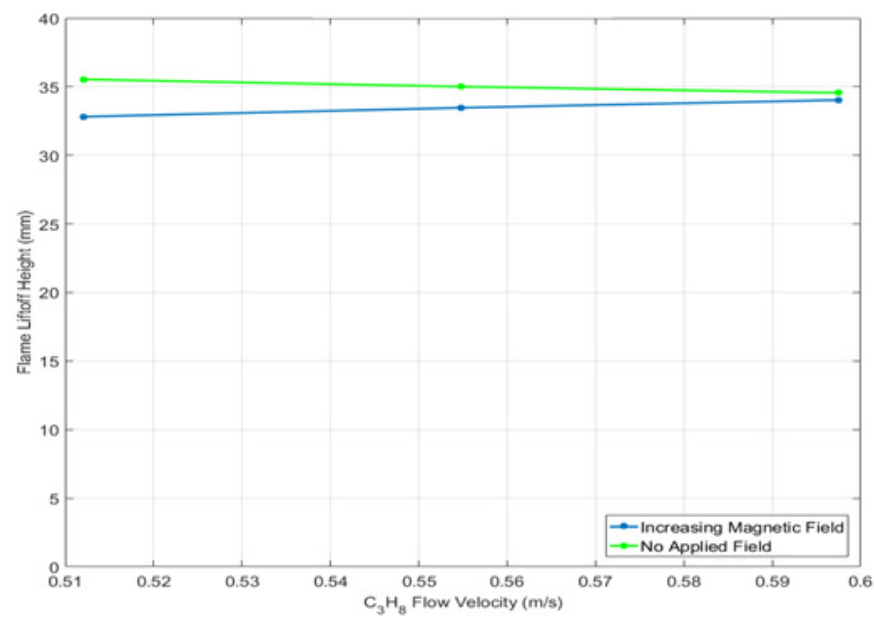

Figure 9 Liftoff height comparison for the $0.81 \mathrm{~mm}$ burner.

As a summary, it was observed that the presence of an increasing gradient field caused the flame height to increase, causing the shape to be more elongated and slender while the flame became shorter under the influence of a decreasing gradient field. Their variation is plotted along a flame whose height is measured in the absence of a magnetic field. Wakayama ${ }^{7}$ explained that this observation may be attributed to the paramagnetic nature of oxygen and the diamagnetic nature of the combustion products which cause a promotion of the combustion reaction when the fuel gas experiences a decreasing gradient field and the reverse effects in an increasing magnetic field. These observations suggest the possibility that a chemical reaction involving a change in the magnetic susceptibilities of component species could be controlled by application of inhomogeneous magnetic fields. It was observed that the presence of gradient field caused the flame liftoff height to decrease irrespective of the direction of the gradient field. The observations can be dissociated into two effects due to the yokethe aerodynamics effect and the magnetic effect. The lift is appeared to be reduced by both these effects. The attached flame region for the increasing field test was maybe to the presence of the yoke. The variation is plotted along a flame whose liftoff height is measured in the absence of a magnetic field. It can be concluded that the reduction in height due to the application of gradient field appears to be independent of the fuel flow rates. These observations suggest the possibility that a chemical reaction involving a change in the magnetic susceptibilities of component species could be controlled by application of inhomogeneous magnetic fields.

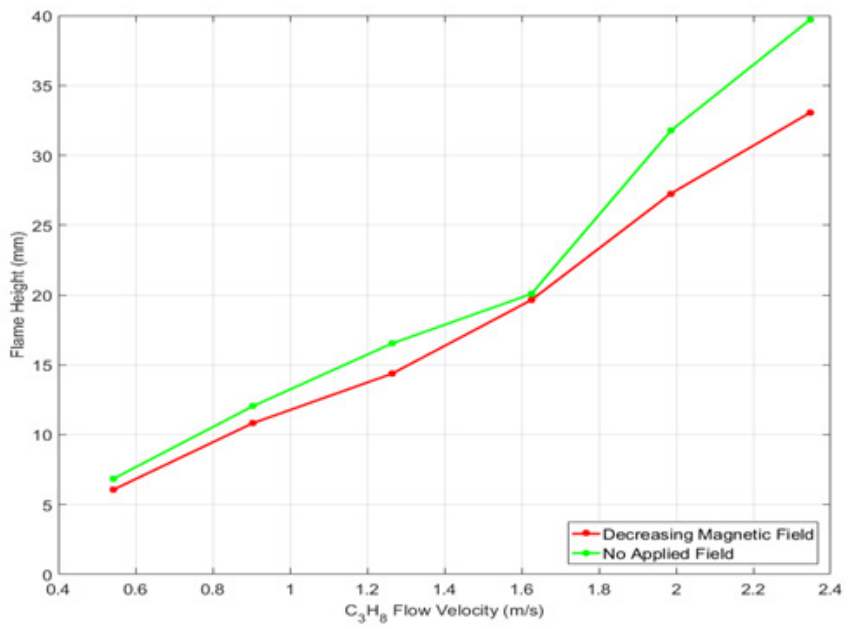

Figure 10 Flame height variation in a decreasing gradient field for the $0.55 \mathrm{~mm}$ burner.

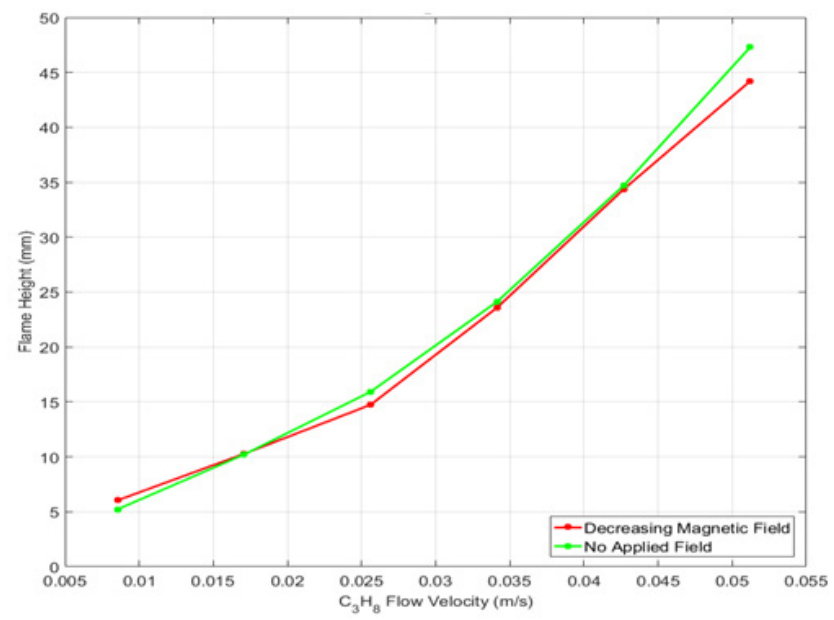

Figure I I Flame height variation in a decreasing gradient field for the $0.8 \mathrm{Imm}$ burner. 


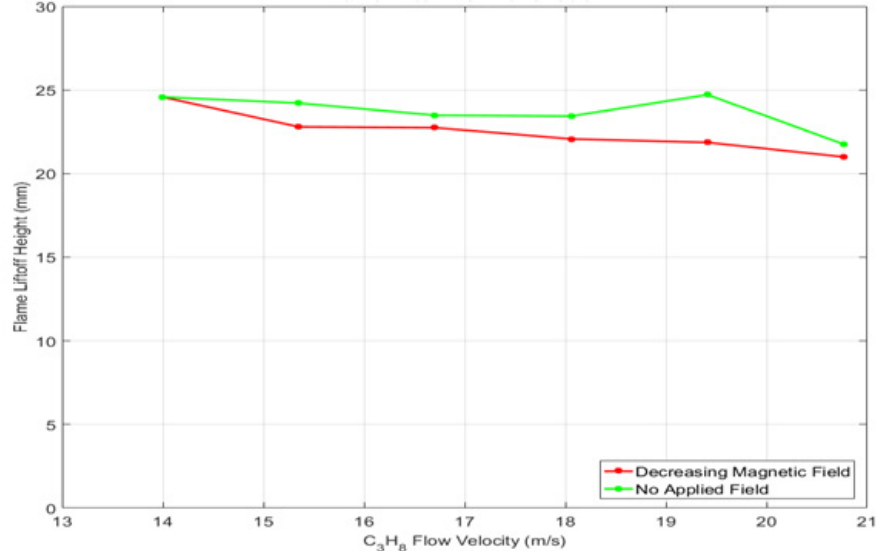

Figure I 2 Liftoff height comparison for the $0.55 \mathrm{~mm}$ burner.

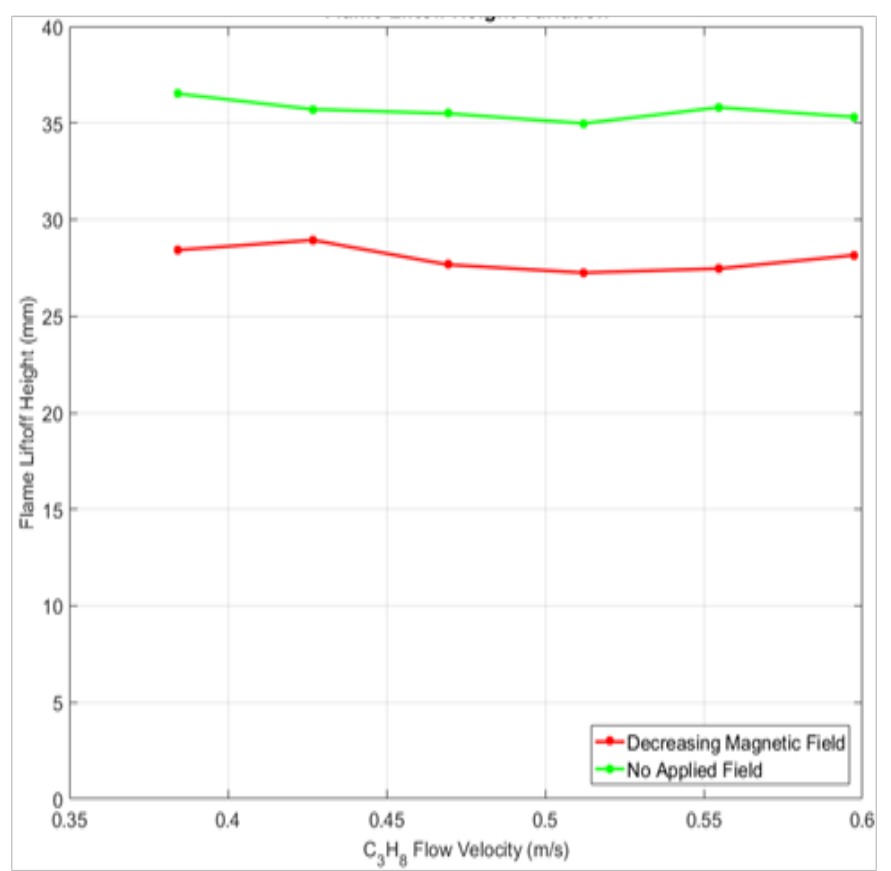

Figure 13 Liftoff height comparison for the $0.81 \mathrm{~mm}$ burner.

\section{Findings and potential utility}

The study of flames and their sensitivity to magnetic fields have been the focus of researchers in the last few years. It has been shown that flames can be manipulated by strong magnetic fields. This study sought to replace the use of electromagnets with permanent magnets that are sufficiently strong enough to bring about a variation in the flame structure and liftoff height of a propane diffusion flame. The observations of this simple, preliminary investigation can be summarized as follows:

a. The height of the flame increased in the presence of an increasing gradient field compared to the no field applied case. This increase in height was found to be dependent on the burner size. b. The height of the flame was reduced in the presence of a decreasing gradient field case compared to the no field applied case. This effect was found to be independent on the burner size although it was more pronounced on the $0.55 \mathrm{~mm}$ diameter burner.

c. A decrease in the liftoff height was observed for both the burners under the influence of a decreasing gradient field. The reduction in liftoff height appeared to be independent of the fuel flow rates. For the $0.81 \mathrm{~mm}$ burner, low flow rates caused the flame to attach to the burner lip. This may be due to the aerodynamic effect of the yoke.

There is potential for future investigations that can be conducted in this area to further understand the complex nature of the interactions between magnetic fields and reaction zones in flames.

i. The current investigation uses a yoke with magnets mounted on the axis of the burner. Future experiments can investigate the impact of the orientation of the magnets on the flame and gas flow.

ii. Flame structure and liftoff height variations can also be investigated in the presence of both electric and magnetic fields.

This phenomenological experiment has demonstrated the potential for increasing magnetic fields to greatly impact flame stability, and future work is warranted, especially as stronger magnets are becoming readily available at low cost.

\section{Acknowledgements}

The work supported in this preliminary paper has been partially supported by the U.S Army Research Office (Contract W911NF1610087) Dr. Ralph Anthenien, Technical Monitor, ARO.

\section{Conflict of interest}

Author declares that there is no conflict of interest.

\section{References}

1. Lawton J, Weinberg FJ. Electrical Aspects of Combustion. USA: Clarendon Press; 1969. p. 1-368.

2. Faraday M. On the Diamagnetic Conditions of Flame and Gases. The London, Edinburgh and Dublin Philosophical Magazine and Journal of Science. 1847;31(210):401-421.

3. Ueno S. Quenching of Flames by Magnetic Fields. Journal of Applied Physics. 1989;65:1243-1245.

4. Mizutani Y, Fuchihata M, Ohkura Y. Pre-Mixed Laminar Flames in a Uniform Magnetic Field. Combustion and Flame. 2001;125(1-2):1071-1073.

5. Gillon P, Blanchard J, Gilard V. Methane/Air-Lifted Flames in Magnetic Gradients. Combustion Science and Technology. 2010;182(11-12):1805-1819.

6. Turns SR. An Introduction to Combustion. McGraw Hill Education, USA; 2012. p. 1-752.

7. Wakayama NI. Effect of a Gradient Magnetic Field on the Combustion Reaction of Methane in Air. Chemical Physics Letters. 1992;188(3-4):279-281. 